\title{
A TEXT-MINING ANALYSIS OF ONLINE REVIEWS ON CAR-SHARING SERVICES
}

\author{
Yun Jik Jeong, Yeon Ji Yang, Jaehye Suk and Kee Ok Kim \\ Department of Consumer \& Family Sciences, Sungkyunkwan University, Korea \\ 25-2, Sungkyunkwan-ro, Jongno-gu, Seoul, Republic of Korea
}

\begin{abstract}
Two of the key challenges of the 21 st century are the sustainable use of natural resources and global climate protection. The car-sharing service has emerged as an alternative to vehicle ownership and can contribute to addressing the economic and environmental challenges posed by mobility. However, for this to happen, consumers must be willing to use car-sharing services, which need to develop a consumer-driven perspective. This study explores consumers' perspectives by investigating online reviews on car-sharing in google play website. A text-mining analysis of online reviews in Korea was conducted. Four topic-modeling keywords on car-sharing services were analyzed with text data regarding the last three years. Understanding chances and risks as well as advantages and disadvantages from consumers' perspectives aids the planning and designing of a better car-sharing service. This study reveals grass-root opinions from consumers and implies that car-sharing service providers have to meet market demands in order to increase market penetration.
\end{abstract}

\section{KEYWORDS}

Car-sharing Service, Text Mining, Online Reviews, Topic Modeling

\section{INTRODUCTION}

The sustainable use of natural resources and global climate protection are two of the key challenges of the $21^{\text {st }}$ century. The automotive sector is seen as a major contributor of environmental impact. As a possible solution to this problem, the sharing economy which accentuates the new concept of sharing-instead-of-owning, has emerged and spread rapidly (Schade, Krail, \& Kuhn, 2014).

Car-sharing services are one of the most successful models of sharing economies. According to Global Market Insights (2018), the global car-sharing market was valued at over \$1.5 billion in 2017 and is predicted to grow to $\$ 11$ billion by 2024 . Car-sharing denotes the shared use of passenger cars, organized by a special supplier (Almeida, Silva, \& Leite, 2017). It indicates positive environmental impacts, such as improved air quality, and offers solutions to traffic-related problems, such as congestion and limited parking spaces.

For car-sharing services to penetrate the Korean market, the willingness to use these services must exist in the population. This requires a consumer-driven perspective and knowledge about the attitudes of consumers toward car-sharing. To understand consumers' perspectives, this study conducted a text-mining analysis of online reviews of SOCAR, which is the most representative car-sharing service company in Korea.

\section{METHOD}

Text data was collected from online reviews of SOCAR posted on the Google Play website from January 1, 2016 to October 21, 2018 by using the R 3.4.3 version. The final dataset was composed of 3,914 reviews. A text-mining analysis was conducted to extract the ten most frequently used keywords and to classify the reviews into four topics with the "topic models" package in the R 3.4.3 version. Topic modeling algorithms are used to discover a set of topics from a large collection of documents, where a topic is a distribution of terms that are biased around those associated under a single theme (Blei \& Lafferty, 2006). This study extracted four topics revealing consumers' opinions of the car-sharing service, SOCAR. 


\section{RESULTS}

The findings are as follows. First, the number of online reviews of car-sharing services has increased from 495 in 2016 to 2,142 in 2018. Second, as shown in Figure 1, the most frequently used keyword associated with car-sharing services is "convenience," followed by "reasonable price," "registration process," and "vehicle management." This indicates that a key factor for the success of the car-sharing service is the ease of use that comes from being able to pick up and return a vehicle round the clock at a reasonable cost. Third, four topics, titled "benefits of using," "purposeful use," "usage shortcomings," and "discomfort of use procedure," are extracted, as shown in Table 1.

The first topic, "benefits of using" includes keywords such as vehicle types, return places, coupons, and convenience. This indicates that a wider availability of vehicles and a better integration with public transports for ease of travel, in combination with reduced cost due to coupons, are fundamental for the growth of the service. The second topic, "purposeful use" contains keywords such as convenience, driving, usefulness, and with girlfriend. Consumers seem to utilize the car-sharing service purely for the purpose of traveling and for special occasions like dating. The third topic, "usage shortcomings" contains keywords such as expensive, inaccurate treatment, unkindness, and local discrimination. These shortcomings imply that the steps that need to be taken before people can access a car still require some fine-tuning using a human-centered perspective. The fourth topic, "discomfort of use procedure" contains keywords such as complex sign-up, deleted, credit card information, and severe battery exhaustion. This implies that car-sharing services need to offer extremely convenient and intelligent ways to make the registration process seamless.

Understanding the advantages and disadvantages as well as chances and risks from consumers' perspectives helps to plan and design a better car-sharing service. These results reveal the grass-root opinions from consumers and imply that car-sharing service providers have to meet market demands in order to increase market penetration.

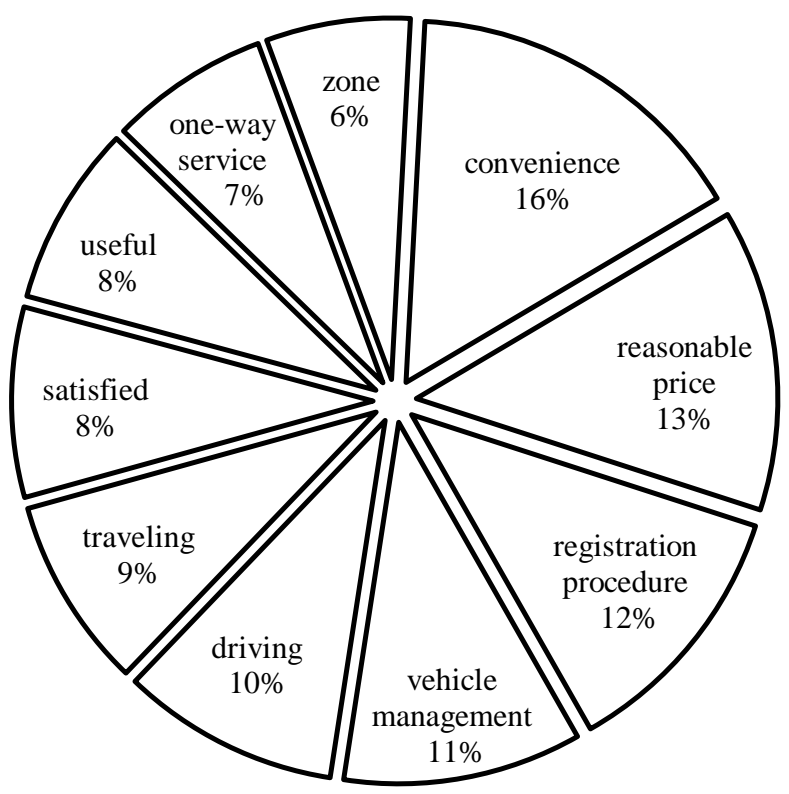

Figure 1. Top ten keywords on car-sharing services 
Table 1. Results of a topic modeling

\begin{tabular}{lllll}
\hline \multirow{2}{*}{ Rank } & Advantages & & Disadvantages & \\
\cline { 2 - 5 } & Benefits of using & Purposeful use & Usage shortcomings & Discomfort of use procedure \\
\hline 1 & vehicle types & convenience & expensive & complex sign-up \\
\hline 2 & return places & driving & inaccurate treatment & deleted \\
\hline 3 & coupons & usefulness & unkindness & credit card information \\
\hline 4 & convenience & with girlfriend & local discrimination & severe battery exhaustion \\
\hline 5 & clean vehicle & travel & full return location & frequent updates \\
\hline 6 & smart key & reliving stress & penalty & difficult to log in \\
\hline 7 & vehicle maintenance & business trip & smart key malfunction & reservation difficulty \\
\hline 8 & useful & recommend & expensive long-distance & return error \\
\hline 9 & delivery service & coupons & customer service & delayed navigation updates \\
\hline 10 & reasonable price & dating & connection & registration error \\
\hline
\end{tabular}

\section{CONCLUSION}

Car-sharing services have emerged as alternatives to vehicle ownership and can contribute to address the economic and environmental challenges posed by mobility. However, for this to happen, consumers must be willing to use car-sharing services, which need to develop a consumer-driven perspective. The results of this study demonstrate that consumers using car-sharing services tend to be satisfied by the type and level of maintenance of the vehicles. In addition, consumers use car-sharing services for traveling, relieving stresses, or driving with girlfriends, which implies that many consumers are inclined to use the service for special occasions like a business trip to a local area and dating. This study reveals that car-sharing services have not yet fully penetrated consumers' daily lives and seem to be regarded as a special platform.

On the other hand, dissatisfied consumers have not only tended to value the post-processing phase but have also reacted sensitively to the sharing of personal information during the registration process. Since car-sharing services are still at a nascent stage in Korea, it is necessary to supplement the advantages and disadvantages found in this study. Further research from consumers' perspectives will reinforce consumers' faith in the platform and assure deeper penetration of car-sharing services in the Korean market.

\section{REFERENCES}

Almeida, F. et al, 2017. Proposal of a Carsharing System to Improve Urban Mobility, Theoretical and Empirical Researches in Urban Management, Vol. 12, No. 3, pp 32-44.

Blei, D. M. and Lafferty, J. D., 2006. Dynamic topic models. In Proceeding of the $23^{\text {rd }}$ international conference on Machine learning. Pittsburgh, PA, USA. pp 113-120.

Global Market Insights, 2018. Car Sharing Market worth over $\$ 11$ bn by 2024. [On-line]. Available: www.gminsights.com/pressrelease/carsharing-market.

Schade, W. et al, 2014. New Mobility Concepts: Myth or Emerging Reality? In Transport Research Arena (TRA) 5th Conference: Transport Solutions from Research to Deployment. Paris, France. 